http://jmscr.igmpublication.org/home/ ISSN (e)-2347-176x ISSN (p) 2455-0450 crossref DOI: https://dx.doi.org/10.18535/jmscr/v7i10.12

\title{
Bilateral Simultaneous Central Retinal Vein Occlusion with no other Systemic Association: Case Report
}

\author{
Authors \\ Mousumi Malakar', Kiran Bhanot ${ }^{2}$, Nawab Zishan Farooqui ${ }^{3}$
}

\begin{abstract}
Central retinal vein occlusion (CRVO) is a common cause of vision impairment which can occur at any given age. Most of the cases are unilateral; bilateral cases are rare, usually having an underlying systemic illness. We report a rare challenging case of bilateral simultaneous central retinal vein occlusion (CRVO) without association of any systemic disease. A 60 year-old Asian Indian female presented with complaints of sudden painless diminution of vision in the right eye followed by left eye. There was no history of any other ocular complaints, trauma, or any ocular intervention. The systemic history was unremarkable. Anterior segment examination revealed a clear cornea with dilated fixed pupil, neovascularization of iris in right eye and semi dilated sluggish reacting pupil in left eye. Fundoscopy showed right eye media hazy due to resolving vitreous hemorrhages, vessels were dilated and tortuous with multiple flame-shaped hemorrhages in all four quadrants of both eyes and vascular sheathing in left eye. The macula was edematous. Fundus fluoresce in angiography revealed perfusion only in peripapillary area suggestive of old retinal vessels occlusion, scattered retinal hemorrhages and venous tortuosity with macular edema bilaterally. This case reveals the occurrence of simultaneous bilateral central retinal vein occlusion and not associated with any systemic disease, it is rare and very challenging case not responding to any treatment with rapid progression inspite of immediate PRP and anti-VEGF injection.

Keywords: Central retinal vein occlusion, Macular edema, fundus fluorescence angiography.
\end{abstract}

\section{Introduction}

Central retinal vein occlusion (CRVO) is a painless loss of vision that can be caused by a swollen optic disc, by dilated retinal veins, and by retinal hemorrhages, is also called venous stasis retinopathy. Central retinal vein occlusion (CRVO) is a common cause of vision impairment which can occur at any given age. Most of the cases are unilateral; bilateral cases are rare, usually having an underlying systemic illness. The reported prevalence rate of bilateral CRVO is $0.41 \%$ to $7.7 \%$ of all CRVO cases ${ }^{1}$. We report a rare challenging case of bilateral simultaneous central retinal vein occlusion (CRVO) without association of any systemic disease.

\section{Case Report}

A 60-year-old female presented with complaints of sudden painless diminution of vision in the right eye followed by left eye. There was no history of any other ocular complaints, trauma, or any ocular intervention. The systemic history (diabetes, hypertension, heart diseases, any other) was unremarkable. There was no history of bleeding tendencies, fainting episodes, and malnutrition or drug intake. On examination, the best corrected visual acuity was HM in right eye and $4 / 60$ in left eye. Anterior segment 
examination revealed a clear cornea with dilated fixed pupil, and semi dilated sluggish reacting pupil in left eye. On slit lamp neovascularization of iris in right eye The intraocular pressure was measured to be $32 \mathrm{mmhg}$ (RE) and $12 \mathrm{mmhg}$ (LE). Fundoscopy showed right eye media hazy due to resolving vitreous hemorrhages, vessels were dilated and tortuous with multiple flameshaped hemorrhages in all four quadrants of both eyes and vascular sheathing in left eye. The macula was edematous. Fundus fluorescein angiography revealed perfusion only in peripheral area suggestive of old retinal vessels occlusion (figure 1). Optical coherence tomography (OCT) showed macular edema in left eye with foveal thickness of $204 \mu$ (RE) and $264 \mu$ (LE) in figure 2 In our patient, laboratory tests including complete blood count, blood sugar, lipid profile and liver and renal function tests and coagulation profile (PT, INR) were within normal range. The rheumatic factor, antinuclear antibodies and antineutrophil cytoplasmicantibodies (pANCA, cANCA), serum homocystine was also normal. Physician reference was done to rule out any neurological deficit, we could not find any abnormality on Doppler evaluation of the carotid artery or echography of the heart, which can rule out those sites as the source of embolism. Indeed the absence of an embolus in the retinal artery means the occlusion was not caused by this.

The patient was treated with intravitreal injection of bevacizumab $(1.25 \mathrm{mg} / 0.05 \mathrm{ml})$ followed by Pan Retinal Photocoagulation (Figure 3), and eye drop brimonidine, timolol and dorzolamide. Intraocular pressure reduced.

Table 1: Results of some laboratory test

\begin{tabular}{|l|c|}
\hline Tests & Results \\
\hline Complete Blood Count & \\
Hemoglobin & $9.1 \mathrm{gm} / \mathrm{dl}$ \\
TLC & $6300 / \mathrm{cumm}$ \\
Differential Leucocyte Count & \\
Neutrophil & $54 \%$ \\
lymphocyte & $24 \%$ \\
eosinophil & $06 \%$ \\
monocyte & $02 \%$ \\
basophil & 00 \\
\hline
\end{tabular}

\begin{tabular}{|l|c|}
\hline P.C.V & $29.7 \%$ \\
M.C.V & $73.4 \mathrm{fl}$ \\
M.C.H & $23.7 \mathrm{pg}$ \\
M.C.H.C & $17.9 \mathrm{gm} / \mathrm{dl}$ \\
RBC & $3.98 \mathrm{mil} / \mathrm{cumm}$ \\
PLATELET COUNT & $1.9 \mathrm{lakh} / \mathrm{cumm}$ \\
ESR & $23 \mathrm{~mm} / \mathrm{hr}$ \\
\hline Peripheral smear & \\
Normocytic normochromic anemia, & \\
No haemoparasite seen & \\
\hline
\end{tabular}
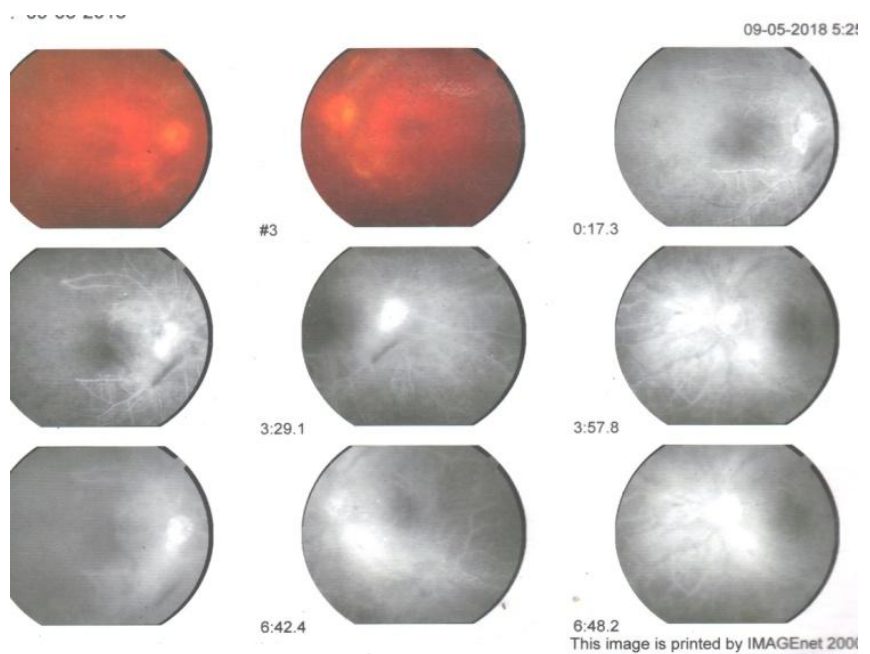

Figure 1: FFA showed perfusion only in peripheral area.

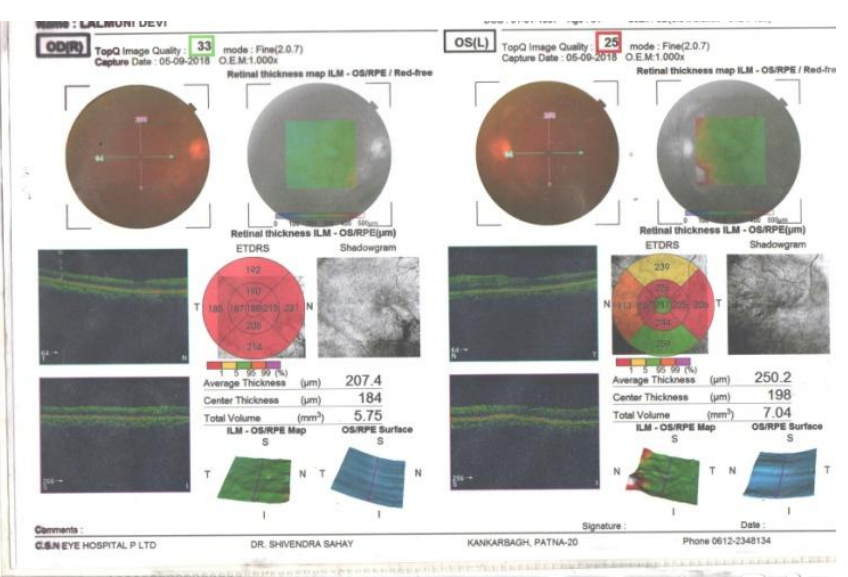

Figure 2: OCT showed macular edema.

\section{Discussion}

Central retinal vein occlusion is a common cause of sudden unilateral loss of vision in elderly. It is usually a unilateral phenomenon presenting in more than $90 \%$ of cases as unilateral sudden loss of vision. The reported prevalence rate of bilateral CRVO is $0.41 \%$ to $7.7 \%$ of all CRVO cases ${ }^{1}$. Only in less than $10 \%$ of cases it presents as bilateral disease ${ }^{2}$, usually having an underlying systemic illness such as a hyperviscosity syndrome or an inflammatory condition ${ }^{3,4,5,6}$ and 
simultaneous presentation is even more rare $^{2}$. However, when it occurs bilaterally it can be life altering. This patient's visual prognosis was poor from the start considering the entering visual acuities, the amount of ischemia, and gross macular edema. Common risk factors include systemic diseases like age related atherosclerosis, hypertension, Diabetes mellitus, hyperlipidaemia, blood dyscrasias, clotting disorders and autoimmune disorders. Local factors like raised intra cranial pressure, vasculitis of retinal vein, closed-head trauma, primary open-angle glaucoma or angle-closure glaucoma and congenital anomaly of retinal vein.

Clinically it presents in two forms i.e. non ischemic and ischemic variety. The non-ischemic type carries good prognosis while the ischemic type carries poor prognosis ${ }^{7}$. Central retinal vein occlusion (CRVO) is one of the most dramatic clinical events in Ophthalmology for which there is usually no treatment. Most interventions are aimed at the prevention or treatment of complications. $^{8}$

CRVO has a better prognosis in young people. In older patients who receive no treatment, about one third improve their own, about one-third wax and wane and stay about same, and about one third get worse. If there is macular edema, it may improve its own. In patients with CRVO, vascular endothelial growth factor (VEGF) is elevated; this leads to swelling as well as new vessels that are prone to bleeding. The most common treatment, based on results from powerful randomized clinical trials, involves periodic injections into the eye of an anti-VEGF drug to reduce the new blood vessel growth and swelling. Patients with ischemic CRVO have worse vision with less chance for improvement. They have a tendency for the eye to cause new blood vessels to growand in the front of the eye, these new vessels can clog the outflow of normal eye fluids. The eye pressure goes up and glaucoma develops. In the back of the eye, news vessels may cause bleeding ${ }^{9}$.

Management of a central retinal vein occlusion, regardless of the etiology, is determined by whether the occlusion is ischemic or non ischemic. Risk factors for developing neovascular iris in patients with CRVO are the amount of nonperfused retina, extent of retinal hemorrhages and central vein occlusion of less than one month duration. The central vein occlusion study (CVOS) data did not support the recommendation of pan retinal photocoagulation (PRP). The CVOS found that early PRP decreased the rate of iris neovascularization; moreover, the study showed that early PRP reduced but did not eliminate the possibility of anterior segment neovascularization. The CVOS recommended close follow-up of eyes with CRVO during the first 6 months (including gonioscopy and undilated slit lamp examination of iris) and prompt PRP of eyes in which iris neovascularization /angle neovascularization develops ${ }^{10}$.

This case reveals the occurrence of simultaneous bilateral central retinal vein occlusion and not associated with any systemic disease, it is rare and very challenging case not responding to any treatment with rapid progression inspite of immediate pan retinal photocoagulation and antiVEGF injection.

\section{References}

1. Bilateral central retinal vein occlusion in multiple myeloma: Mohammed Ziaei, Mostafa A. Elgohary, Fion D. Bremner CAN J Ophthalmol-Vol. 48, No. 2, April 2013

2. Dr Purabi Rani Debnath, Associate Professor Department of Ophthalmology, BIRDEM General Hospital, Dhaka.

3. Hayreh SS, Zimmerman B, McCanthymj, systemic diseases associated with various types of retinal vein occlusion. Am j ophthalmol.2001;131:61-77

4. Gelman R, DiMango EA, Schiff WM. Sequential bilateral central retinal vein occlusions in a cystic fibrosis patient with hyperhomocysteinemia and hypergammaglobulinemia. Retin Cases Brief Rep. 2013; 7:362-7. 
5. Aggio FB, Cariello AJ, Almeida MS, Rodrigues CA, De Moraes NS, Colleoni $\mathrm{GW}$, et al. Bilateral central retinal vein occlusion associated with multiple myeloma. Ophthalmologica. 2004; 218:283-7.

6. Tseng MY, Chen YC, Lin YY, Chu SJ, Tsai SH. Simultaneous bilateral central retinal vein occlusion as the initial presentation of acute myeloid leukemia. Am J Med Sci. 2010;339: 3879.

7. Hayreh SS (1976) So-called 'central retinal vein occlusion', Venous stasis retinopathy. Ophthalmologica 172:14-37.

8. $\mathrm{P}$ Alexander, D Flanegar, $M$ Hingorani "Bilateral simultenous CRVO secondary to hyperviscosity in waldenstroms macroglobu-linaemia" Eye22,10891092(2008)

9. ASRS: The foundation of American society of retina specialists, Retina health series (312) 578-8760

10. The central vein occlusion study group: A randomized clinical trial of early panretinal photocoagulation for ischemic central vein occlusion; ophthalmology. 1995;102:1434-1444. 